\title{
La retroalimentación constructiva en el desarrollo de habilidades comunicativas escritas e investigativas en dos generaciones de alumnos de medicina en Yucatán, México
}

\author{
Constructive feedback in the development of written and research communicational \\ skills in two generations of medical students in Yucatan, Mexico
}

\author{
Esteban Aguilar-Vargas ${ }^{1}$, Adriana Rodríguez-Castellanos², Luis Baeza², \\ Nina Méndez ${ }^{4}$ \\ Estudiante de quinto año de la carrera de médico cirujano, Campus de Ciencias de la Salud, Universidad Marista de Mérida, Yucatán, \\ México. \\ ${ }^{2}$ Médico Cirujano, Estudiante de la Maestría en Investigación en salud, posgrado Institucional en Ciencias de la Salud, Universidad \\ Autónoma de Yucatán, México. \\ ${ }^{3}$ Médico cirujano, Hospital Regional de Alta Especialidad de la Península de Yucatán (HRAEPY), México. \\ ${ }^{4}$ Doctora en Ciencias de la Salud, Maestra en Ciencias, Médico Cirujano; Profesora Titular, Campus de Ciencias de la Salud, Universidad \\ Marista de Mérida, Yucatán, México.
}

\begin{abstract}
Resumen
Antecedentes. La generación de competencias para la investigación en medicina es fundamental para que los médicos apliquen las habilidades científicas en su quehacer diario; los programas académicos que incluyen la formación de habilidades investigativas requieren a su vez habilidades para la comunicación escrita en los alumnos. La retroalimentación constructiva es un medio comúnmente empleado en medicina, por el cual el docente acompaña al alumno, dándole a conocer sus fortalezas, debilidades y oportunidades para un mejor desempeño, mientras asegura una evaluación justa. A pesar de ello, su aplicación más difundida es en la práctica clínica y no en el aprendizaje de la redacción y comunicación científica. Objetivo. Evaluar el impacto de la retroalimentación constructiva en el desempeño de los estudiantes en sus propuestas de protocolos clínicos y epidemiológicos considerando aspectos de redacción y metodológicos. Diseño. Estudio analítico, prospectivo. Lugar. Universidad Marista de Mérida, Yucatán, México. Participantes. Alumnos regulares que cursaron la materia Métodos Clínicos y Epidemiológicos de la carrera de Médico Cirujano durante los ciclos escolares 2014-2015 y 2015-2016. Intervención. Se evaluaron los puntajes obtenidos en el protocolo de investigación antes y después de la retroalimentación constructiva. Resultados. La media de la puntuación inicial de los protocolos fue $8,3 \pm 0,8$ y la media posterior a la retroalimentación constructiva fue 9,4 $\pm 0,9$; el incremento de las puntuaciones en promedio fue de 1,1 $\pm 0,1$ con un valor de $t=7,70$ $p=0,000$. Las habilidades para la comunicación escrita que mejoraron los alumnos fueron fundamentalmente: 1 . El manejo del tema, 2. La organización del texto, 3. Empleo del lenguaje escrito, redacción, y 4. Fuentes de información; los alumnos presentan todas las ideas no propias en su escrito con las citas a la fuente de donde fueron extraídas. Conclusión. El uso de la retroalimentación constructiva mostró una mejora significativa en el puntaje obtenido por los alumnos en su protocolo de investigación.
\end{abstract}

Palabras clave. Aprendizaje; Educación Médica; Retroalimentación Constructiva; Investigación; Pregrado.

\section{Abstract}

Background. Research and written communication skills are essential in undergraduate medical education, because medical doctors are often involved in research, and written communication is part of a medical doctor's everyday routine. Academic medical programs that include training in clinical or epidemiological research require written communication aptitudes. Constructive feedback is a review technique commonly employed in medical education that involves the professor's close surveillance of the student's performance and a clear communication to respectfully explain to students their strengths, weaknesses and opportunities of improvement, while ensuring a fair evaluation. Even though constructive feedback is common in clinical practice, it is rarely used in undergraduate medical training in scientific writing. Objective. To evaluate the impact of constructive feedback on the academic performance of students in their clinical or epidemiological research protocol proposals, involving written and methodological aspects. Design. Analytical, prospective study. Setting. Universidad Marista de Mérida, Yucatán, México. Participants. Students in their fourth year of medical education. Intervention. The quantitative evaluations obtained in the research protocol proposals before and after the constructive feedback were compared in 103 students in their fourth year of medical education who were attending the Clinical and Epidemiological Research Methods course during 2014-2015 and 2015-2016 school years. Results. The mean of the initial score was $8.3 \pm 0.8$, and the mean post constructive feedback was $9.4 \pm 0.9$; the average score increase was $1.1 \pm 0.10$ with $t=7.70$ value, $p=0.000$. Students improved the following communication skills: 1. Theme presentation and review, 2. Organization of a scientific manuscript, 3. Use of written language, and 4. Use of information sources. Conclusion. There was a significant improvement in protocol proposals following provision of constructive feedback reviews to students, both in written and methodological aspects.

Keywords. Learning; Medical Education; Constructive Feedback; Research; Undergraduate Students.

An Fac med. 2016;77(2):137-42 / http://dx.doi.org/10.15381/anales.v77i2.11818 


\section{INTRODUCCIÓN}

Desarrollar y fortalecer las habilidades investigativas de los médicos en formación es tan importante como la capacitación clínica, pues es un anhelo que como profesionistas tengan la capacidad para analizar críticamente las acciones preventivas, diagnósticas o terapéuticas de elección en su práctica diaria y es por ello que es deseable que los estudiantes de medicina participen en la investigación y adicionalmente, si desean en un futuro participar en la generación de conocimientos, las habilidades para la investigación y la comunicación escrita serán fundamentes para cumplir dicho fin ${ }^{(1)}$.

Perfeccionar las habilidades para la comunicación escrita es una necesidad fundamental de la formación profesional, ya que la capacidad de comunicarse apropiadamente de manera oral y escrita es sin duda una de las competencias básicas que hacen posible dar a conocer con claridad y estructura los pensamientos y opiniones. Al médico en formación le sirve para expresarse en el ámbito clínico (tales como el razonamiento diagnóstico, manejo de casos, y en general comunicación con colegas y pacientes), a la vez que también facilita las destrezas de la comunicación científica derivada de la investigación biomédica y de las ciencias de la salud. Debido a ello, en la actualidad se reconoce que las habilidades para la comunicación son aspectos susceptibles a enseñarse, aprenderse y evaluarse, aún cuando la introducción de estas capacidades en la formación de pregrado en medicina es relativamente reciente ${ }^{(2-4)}$.

Más allá de la comunicación per se, para lograr habilidades investigativas es importante que los médicos en formación posean destreza para transmitir una correcta estructura del pensamiento, claridad con el estilo de escribir en el arreglo espacial de las palabras, mientras presenten la información escrita sin defectos de ortografía o sintaxis. Para la comunicación científica necesitan además la capacidad de síntesis y congruencia en la redacción de los ele- mentos metodológicos, como lo son los objetivos, las hipótesis o conclusiones. Para ello, la evaluación de protocolos escritos por los estudiantes de pregrado en medicina es una vía útil para evaluar y reforzar las habilidades comunicativas de los médicos en formación, perfeccionar capacidades profesionales críticas y facilitar su razonamiento científico metodológico ${ }^{(5,6)}$.

La comunicación científica e investigación de pregrado en salud es reducida a nivel mundial, particularmente en América Latina, donde se estima que los estudiantes de medicina participan en 4,5\% de las publicaciones en el área de la salud y que México aporta apenas un $0,4 \%$ de las publicaciones médicas científicas; este hecho se vincula con la escasa oferta de cursos formales en investigación en los programas académicos de las carreras de medicina en México. Debe reconocerse que en América Latina la capacitación para la atención terapéutica con fines asistenciales se ve favorecida, en detrimento del tiempo y recursos destinados para la formación en investigación y, por ende, de la comunicación científica. Es por ello que, ante la reducida instrucción para la comunicación, se obtiene como consecuencia la baja producción científica en biomedicina en América Latina. El reforzamiento de los programas académicos en las áreas de comunicación escrita y comunicación de la ciencia podría fortalecer las habilidades investigativas e incrementar las publicaciones científicas en México y América Latina, contribuyendo a fortalecer a las ciencias de la salud, la medicina basada en evidencias y la producción científica en la región ${ }^{(7-10)}$.

La evaluación de las habilidades de comunicación e investigativas suele requerir de un acompañamiento constante del desempeño del alumno de pregrado, de modo que una sola evaluación para definir la puntuación o calificación del alumno suele resultar insuficiente. Las competencias para la comunicación e investigación pueden abordarse mediante la evaluación formativa, la cual se aplica como una estrategia para vigilar el progreso del aprendizaje, mientras se proporciona retroalimentación al alumno sobre los logros, fortalezas, debilidades y oportunidades de mejora ${ }^{(11)}$.

Por su parte, la retroalimentación provee al estudiante de reflexión sobre sus actos y consecuencias, le permite llegar a las metas y objetivos y se considera un medio para fortalecer el proceso de aprendizaje y desempeño docente ${ }^{(12)}$. En comparación, la retroalimentación constructiva es una técnica reconocida de evaluación formativa de amplio potencial para su empleo e implementación en medicina, que se caracteriza por ser 1 . Pertinente, 2 . Inmediata, 3. Factual, 4. Útil, 5. Confidencial, 6. Respetuosa, 7. Adaptada, y 8. Alentadora ${ }^{(13)}$.

El empleo de la retroalimentación constructiva en medicina es amplio y se extiende a diferentes áreas de la formación del médico cirujano ${ }^{(11)}$.

En el presente estudio proporcionamos los resultados obtenidos mediante la retroalimentación constructiva, tomando en cuenta la diferencia entre la evaluación inicial y la evaluación final. El objetivo de nuestro estudio consiste en comparar los puntajes obtenidos en las propuestas de protocolos de investigación antes y después de la retroalimentación constructiva, en el marco de la materia de métodos clínicos y epidemiológicos impartida en el séptimo semestre de la licenciatura en medicina.

\section{METODOLOGÍA}

La materia Métodos Clínicos y Epidemiológicos (MCE) forma parte de los cursos obligatorios de la carrera de Médico Cirujano que se imparte en un programa de doce semestres en el campus de ciencias de la salud de la Universidad Marista de Mérida, Yucatán, México. La materia de MCE es consecutiva a la de metodología de la investigación, siendo en MCE donde los alumnos trabajan su primera propuesta de investigación (protocolo) centrada 
en la metodología clínica o la metodología epidemiológica.

La materia MCE involucra la elaboración de un protocolo de investigación en salud empleando los métodos cualitativos o cuantitativos mediante diseños clínicos o epidemiológicos. Desde un inicio, fue del conocimiento de los alumnos que se establecería una fecha límite de entrega previa y de igual forma se establecieron los criterios evaluación tanto de aspectos de forma (estilo, normas de redacción) como de fondo, incluyendo la descripción metodológica de acuerdo a las directrices EQUATOR (Enhancing the QUAlity and Transparency Of health Research, por su nombre en inglés), para estudios clínicos o epidemiológicos. La calificación asignada se evaluó en base a la puntuación alcanzada, empleando la rúbrica 'Expresarse y Comunicarse con Propiedad' (ECP) y las listas de cotejo de las directrices del EQUATOR; los puntajes fueron estandarizados en base a 10 puntos (puntuación máxima). En la materia MCE, la rúbrica de la competencia ECP y las directrices EQUATOR integran una sinergia que permite monitorizar los avances y evaluar el desempeño de los alumnos en sus protocolos de investigación, tanto de fondo como de forma.

Durante los ciclos escolares 2014 2015 y 2015-2016 se llevó registro de las revisiones y la retroalimentación constructiva realizada a los alumnos del séptimo semestre. Esta revisión consistió en que cada alumno elaboró su propuesta de protocolo completa en un archivo Word compartido por medio del Dropbox, de donde el profesor titular o asistente lo recuperarían para revisarlo. Los archivos compartidos debían estar codificados con las iniciales de los alumnos. En cada revisión se consideraron los puntos definidos en la rúbrica ECP y las directrices metodológicas EQUATOR. La retroalimentación constructiva se administró mediante comentarios específicos y notas que involucraron diversos recursos, entre los cuales figuraron ligas externas a artículos científicos en la web, párrafos de libros, tesis, fórmulas estadísticas, definiciones y ejemplos prácticos, todo ello con la finalidad de que el alumno pudiese tener las herramientas de consulta necesarias para realizar los cambios pertinentes, así como la claridad en lo que se solicitaba como parte de la dinámica en la revisión de su protocolo. De tal modo, las sugerencias de cambio por parte de los revisores tenían un fundamento académico-científico que podía ser consultado por el alumno y no correspondían únicamente al estilo individual del profesor. Todo lo anterior se brindaba al alumno con el objetivo de la optimización de los protocolos, siempre de manera positiva mediante sugerencias, como previamente se explica, y nunca de manera negativa a modo de inculcación de ideas o cambios establecidos por el revisor en el trabajo del alumno. Esto pone a manifiesto la meta de la utilización de la retroalimentación constructiva, conceder al alumno una guía para la reflexión de sus habilidades tanto escritas como orales y permitírsele mejorarlas o fortalecerlas. Los alumnos recibieron sus documentos revisados en tiempo real, de modo que pudieron seguir y atender los comentarios de los revisores oportunamente. Debido a que los comentarios se guardaban con cada nueva versión del documento, estos podían ser consultados posteriormente tanto por el alumno como por los profesores. Los profesores titular y asistente se dividieron las revisiones previas a la retroalimentación constructiva al 50\%, luego administraron la retroalimentación constructiva y finalmente se intercambiaron los archivos para la revisión posterior. El tiempo promedio entre revisiones fue de 18 días. Las dudas específicas fueron atendidas en persona con la profesora titular. Los puntajes obtenidos en las versiones previa y posterior a la retroalimentación constructiva fueron tomados en cuenta para efectos de este estudio, como indicadores previos y posteriores a la retroalimentación constructiva ${ }^{(14)}$.

Mediante un proceso de estandarización, el profesor titular de la asignatura y el profesor asistente homologa- ron sus criterios de evaluación con un error máximo de $\pm 0,20$; la asignación de puntuaciones iniciales y finales de las dos generaciones de alumnos fue realizada por un mismo equipo de docentes en el curso. El diseño de asignación de revisores, intercambio y estandarización de las calificaciones se emplearon como medidas para asegurar la calidad y evitar el sesgo. Es conveniente aclarar que los profesores tuvieron conocimiento de los temas que abordarían los alumnos en el semestre, así como del desempeño general de los alumnos en sus calificaciones parciales promediadas, pero la rúbrica de evaluación inicial fue conservada por el profesor que realizó dicha revisión para fines del presente estudio, compartiéndose y analizándose a posteri con el profesor que realizó la segunda revisión. Las rúbricas inicial y final no se vincularon a los protocolos de los alumnos hasta que estos generaron su versión final al término del semestre.

\section{RESULTADOS}

Se incluyó inicialmente a 109 alumnos que cumplieron con los criterios de inclusión (ser alumno regular del séptimo semestre), de los cuales 73 fueron del sexo masculino y 36 del sexo femenino. Un total de seis alumnos fueron eliminados del análisis debido a que, por razones diversas (inasistencias), no se obtuvieron datos sobre la revisión inicial o final de sus protocolos. El tiempo total invertido en las revisiones incluyendo la retroalimentación constructiva fue de 25 minutos por alumno (aproximadamente 22 horas por profesor, entre ambas generaciones, esto es, 44 horas totales). La moda del número de revisiones para los alumnos del cur-

Tabla 1. Distribución de los alumnos por generación y sexo.

\begin{tabular}{cccc} 
Generación & Hombres & Mujeres & Total \\
\hline $2014-2015$ & 28 & 21 & 49 \\
$2015-2016$ & 41 & 13 & 54 \\
\hline Ambas & 69 & 34 & 103 \\
\hline
\end{tabular}




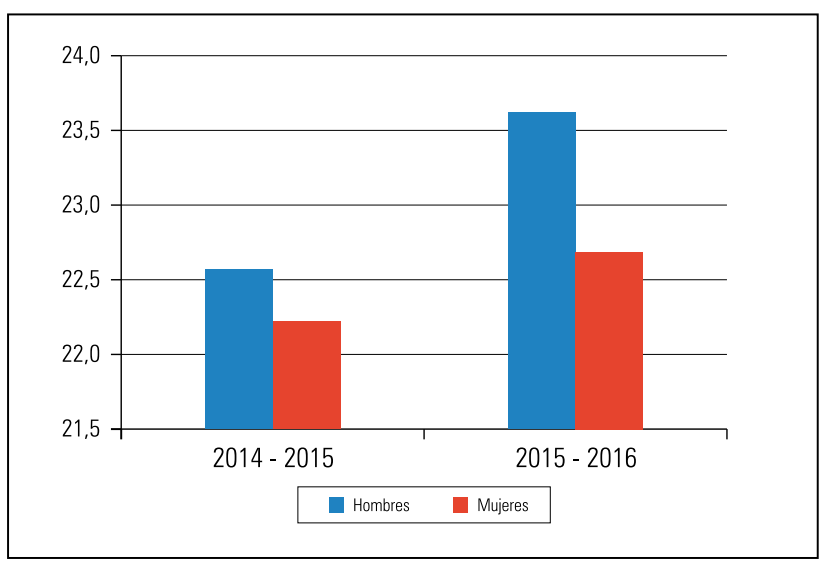

Gráfica 1. Distribución de la media de edad por generación y sexo $(\mathrm{N}=103)$.

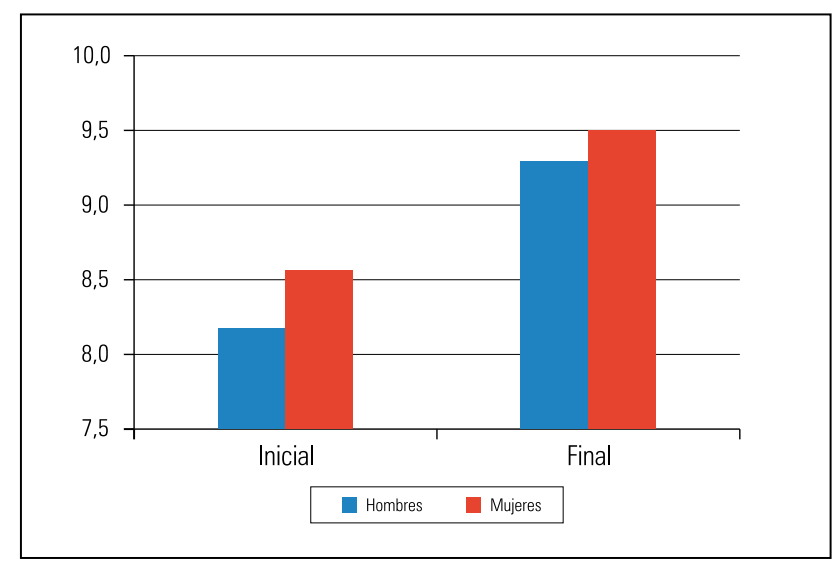

Gráfica 2. Distribución de la calificación media por sexo ( $\mathrm{N}=103)$. so escolar 2014-2015 fue dos y para el 2015-2016 fue tres. En la tabla 1 se presenta la distribución por generación y sexo de la población estudiada y en la gráfica 1 se muestra la distribución por generación edad y sexo. En la tabla 2 se observa la distribución de los alumnos por edad.

La media de puntuación obtenida en las calificaciones iniciales fue 8,31 y la de las calificaciones finales 9,54. No se observó diferencia significativa en las calificaciones iniciales o finales por sexo (en la gráfica 2 se muestra la distribución de calificaciones por sexo), pero sí se observó diferencia significativa en la ganancia de puntuación, obte- niendo una ganancia inicial-final de 0,9 en la generación 2014-2015 y de 1,2 en la generación 2015-2016 ( $p<0,000)$. Cabe mencionar que de los 10 alumnos que no subieron su puntaje, siete de ellos pertenecieron a la generación 2015-2016.

La diferencia final observada en las calificaciones iniciales y finales fue de 1,01 puntos. Se observó diferencia significativa entre la calificación media inicial y final en la prueba $t$ de student para comparación de medias para dos grupos, tal como se muestra en la tabla 3. La información adicional y el repositorio de los 103 archivos previos y 103 posteriores a la retroalimentación cons-

Tabla 2. Distribución de los alumnos por edad.

\begin{tabular}{cccccc} 
Generación & N & Media & D.E. & \multicolumn{2}{c}{ Intervalo de confianza 95\% } \\
\hline $2014-2015$ & 49 & 22,61 & 2,78 & 21,81 & 23,41 \\
$2015-2016$ & 54 & 22,91 & 1,15 & 22,59 & 23,22 \\
\hline Ambas & 103 & 22,76 & 2,09 & 22,35 & 23,22 \\
\hline
\end{tabular}

Tabla 3. Diferencia en la media de calificaciones iniciales (CMI) y finales (CMF), $\mathrm{N}=103$.

\begin{tabular}{lcccc} 
Calificación media & & D.E. & \multicolumn{2}{c}{ Intervalos de confianza 95\% } \\
Inicial & 8,3 & 0,8 & 8,05 & 8,57 \\
Final & 9,4 & 0,9 & 9,27 & 9,54 \\
\hline \multicolumn{1}{c}{$\mathrm{t}=7,70$} & $\mathrm{CMl}<\mathrm{CMF}=1,09$ & $p<0,000$ \\
\hline
\end{tabular}


lle y cuidado en los aspectos relacionados con las consideraciones éticas. No hubo cambios en los temas de investigación inicialmente propuestos, pero sí se cambiaron enfoques, abordajes y metodologías en las propuestas.

\section{DISCUSIÓN}

En este estudio hemos mostrado que la revisión periódica mediante la retroalimentación constructiva con el abordaje de las habilidades para la comunicación escrita además de los aspectos metodológicos, logró mejorar la calidad de los protocolos de investigación de los alumnos de medicina que cursaban el séptimo semestre. El proporcionar a los alumnos revisiones empleando la retroalimentación constructiva permitió: a) reforzar las habilidades de comunicación escrita de los alumnos; b) mejorar las competencias para el planteamiento de protocolos de investigación; y, c) calificar de manera precisa el desempeño del alumno, que como resultado generó que los alumnos obtuvieran mejoría en sus calificaciones atendiendo las revisiones del profesor.

A diferencia de cuando se emplean otros tipos de retroalimentación, como la cognitiva (la cual es vista como un correctivo, donde un experto provee de información a un receptor pasivo), en la retroalimentación constructiva el alumno tiene un papel activo en la retroalimentación, teniendo a través de los comentarios individuales por parte del profesor la oportunidad de compartir experiencias, ganando nuevos conocimientos ${ }^{(17-19)}$. Adicionalmente, la retroalimentación constructiva permite al alumno la oportunidad de mejorar sus calificaciones y por ende, sus promedios, lo cual es importante pues en el área médica los promedios alcanzados en el pregrado pueden ser potencialmente determinantes para la trayectoria académica y laboral futura de los médicos ${ }^{(20)}$.

En estudios previos realizados para evaluar la escritura en estudiantes de medicina y residentes, se ha encon- trado que el empleo de una rúbrica es de utilidad, pues permite dar una retroalimentación estructurada y evaluar intervenciones. En nuestro caso evaluamos las revisiones empleando la retroalimentación constructiva y logramos evaluar cuantitativamente la utilidad de la rúbrica de expresión escrita en combinación con las directrices consolidadas para estudios clínicos y epidemiológicos ${ }^{(21-24)}$.

Desafortunadamente, la retroalimentación constructiva es un proceso continuo que requiere tiempo y dedicación por lo que se considera un componente 'difícil' en la enseñanza médica, por lo que los profesores pueden optar por otros tipos de revisiones y evaluaciones menos complejas ${ }^{(25)}$.

Entre las competencias deseables en un médico cirujano, se espera que sepa cómo escribir, ya sea una nota médica o un escrito académico ${ }^{(21,23,26)}$. Con las bases comprendidas en la competencia de expresión escrita y los contenidos del curso métodos clínicos y epidemiológicos se persigue el fin último de fortalecer la investigación y la comunicación científica de los médicos mexicanos como investigadores productivos.

En México, una intervención desarrollada con la finalidad de estimular la producción científica desarrollada en la Escuela de Medicina del Tecnológico de Monterrey se implementó un curso optativo de verano teórico práctico en el que se generaron como producto ocho proyectos de investigación por equipos, los mismos que fueron presentados en congresos y se hallan en vías de publicación. Mientras en nuestro estudio, que fundamentalmente persigue un fin común, hemos podido monitorizar el desempeño individual de los alumnos, quienes hasta la actualidad han planteado apropiadamente protocolos científicamente rigurosos con pertinencia e impacto social, los mismos que seguiremos en el tiempo para conocer cuántos de ellos finalmente se desarrollarán ${ }^{(27)}$.

A partir de esta primera experiencia desarrollada a lo largo de dos ciclos escolares, nos ha permitido reafirmar la importancia de continuar con las estrategias didácticas empleadas en la materia y continuar con las técnicas de revisión empleadas. Pero, también hemos de reconocer que hará falta ampliar la presencia de los trabajos realizados por los alumnos en los foros científicos especializados.

En este trabajo presentamos únicamente la perspectiva de la puntuación cuantitativa relacionada con los protocolos, sin considerarse las perspectivas y experiencias de los alumnos que participaron, las mismas que podrán ser retomadas en un futuro cercano.

\section{REFERENCIAS BIBLIOGRÁFICAS}

1. Rojas-Redondo V. Las publicaciones en revistas indexadas, único indicador de la producción de las sociedades científicas estudiantiles. Ciencia e Investigación Médica Estudiantil Latinoamericana. 2007;12:5-6.

2. León-Mejía A, Ortiz-Uribe F. La comunicación escrita en el proceso de enseñanza aprendizaje. UPIICSA Repositorio Digital Institucional Instituto Politécnico Nacional. 2005;39(1)

3. Bitran M, Zúñiga D, Flotts P, Padilla O, Moreno R. Mejoría en las habilidades de comunicación escrita de estudiantes de medicina: Impacto de un taller de escritura. Rev Méd Chil. 2009;137:617-34.

4. Clèries X. La esencia de la comunicación en educación médica. Educación médica. 2010;13(1):25-31.

5. Petra-Micu I. La enseñanza de la comunicación en medicina. Inv Ed Med. 2012;1(4):218-24.

6. Cyr P, Smith K, Broyles I, Holt C. Developing, evaluating and validating a scoring rubric for written case reports. International Journal of Medical Education. 2014; 5:18-23. doi: 10.5116/ijme.52c6.d7ef.

7. Ángel-Isaza A, Botero-Suárez H, González D, Piedad-Ospina L, Velasco M, Ocampo M. Interés de los estudiantes de medicina por la investigación. Ciencia e Investigación Médica Estudiantil Latinoamericana. 2010;15(1):9-13.

8. Alarcón-Villaverde J, F FR, Gutiérrez C. Publicaciones científicas estudiantiles producidas en el curso de Epidemiología de la Facultad de Medicina de la Universidad Nacional Mayor de San Marcos durante el periodo 2003-2009. An Fac med. 2010;71(2):111-

9. Chávez-Caraza K, de Ita JR, Ramírez JL, Duarte GV, Lozano-Lee F. Desarrollo e implementación de un curso de investigación para estudiantes de ciencias de la salud: una propuesta para estimular la producción científica. Inv Ed Med. 2015;4(15):1619.

10. Mayta-Tristán P, Cartagena-Klein R, Pereyra-Elias R, Portillo A, Rodriguez-Morales A. Apreciación de estudiantes de Medicina latinoamericanos sobre la capacitación universitaria en investigación científica. Rev Méd Chil. 2013;141:716-22.

11. Evaluación y supervisión en educación médica. Seminario. El ejercicio Actual de la Medicina. [En linea] Disponible en: http://www.facmed.unam. mx/eventos/seam2k1/2006/sep_02_ponencia.html [Internet]. 2010 
12. Vives-Varela $T$, Varela-Ruiz M. Realimentación efectiva. Inv Ed Med. 2013;2(6):112-4.

13. Ovando MN. Constructive feedback: A key to successful teaching and learning. International Journal of Educational Management. 1994;8(6):18-22.

14. Moher D, Altman D, Schulz K, Simera I, Wager E. Guidelines for reporting health research: a user's manual: Wiley; 2014.

15. Small AC, Levy LL. In support of medical student research. Academic Medicine. 2013;88(11):1592. doi: 10.1097/ACM.0b013e3182a7fa30.

16. Zier K, Wyatt C, Muller D. An innovative portfolio of research training programs for medical students. Immunologic research. 2012;54(1-3):286-91. doi: 10.1007/s12026-012-8310-x.

17. Carless D, Salter D, Yang M, Lam J. Developing sustainable feedback practices. Studies in Higher Education. 2011;36(4):395-407.

18. Thurlings M, Vermeulen M, Bastiaens T, Stijnen S. Understanding feedback: A learning theory perspective. Educational Research Review. 2013;9:1-15.

19. Evans C. Making sense of assessment feedback in higher education. Review of educational research. 2013;83(1):70-120.

20. Monterrosas C, Reyes R, Navarrete M, Flores M, Hernández N. Preferencias en la selección de hospital para realizar el Internado Médico en la Carrera de
Medicina. Poster presentado en 2012.

21. Cyr PR, Smith KA, Broyles IL, Holt CT. Developing, evaluating and validating a scoring rubric for written case reports. International journal of medical education. 2014;5:18-23. doi: 10.5116/ijme.52c6.d7ef.

22. Lockspeiser TM, Schmitter PA, Lane JL, Hanson JL, Rosenberg AA, Park YS. Assessing Residents' Written Learning Goals and Goal Writing Skill: Validity Evidence for the Learning Goal Scoring Rubric. Academic Medicine. 2013;88(10):1558-63. doi: 10.1097/ACM.0b013e3182a352e6.

23. Melvin L, Connolly K, Pitre L, Dore K, Wasi P. Improving medical students' written communication skills: design and evaluation of an educational curriculum. Postgrad Med J. 2015 Jun;91(1076):303-8. doi: 10.1136/postgradmedj-2014-132983.

24. E Panadero AJ. The use of scoring rubrics for formative assessment purposes revisited: A review. Educational Research Review. 2013;9(0):129 - 44. doi: http://dx.doi.org/10.1016/j.edurev.2013.01.002.

25. Hewson MG, Little ML. Giving feedback in medical education. Journal of General Internal Medicine. 1998;13(2):111-6.

26. Murdoch-Eaton D, Whittle S. Generic skills in medical education: developing the tools for successful lifelong learning. Medical education. 2012;46(1):120-8.
27. Chávez Caraza KL, Rodriguez de Ita J, Lozano Ramírez JF, Vargas Duarte GM, Lozano Lee FG. Desarrollo e implementación de un curso de investigación para estudiantes de ciencias de la salud: una propuesta para estimular la producción científica. Investigación en Educación Médica. 2015;4(15):161-9. doi: http://dx.doi.org/10.1016/j. riem.2015.04.001.

Artículo recibido el 30 de diciembre de 2015 y aceptado para publicación el 26 de marzo de 2016.

Conflictos de interés:

Ninguno de los autores tiene relación comercial alguna que pueda generar conflictos de interés.

\section{Correspondencia:}

Esteban Aguilar Vargas.

Dirección: Calle 45 número 239 por 20 y 26 diagonal, fraccionamiento Brisas, CP: 97144.

Teléfono: 9999860722 . Celular: 0449992715183.

Correo electrónico: esteban.aguilar.vargass@gmail. com 